\title{
THE SLATER AND SUB- $k$-DOMINATION NUMBER OF A GRAPH WITH APPLICATIONS TO DOMINATION AND $k$-DOMINATION
}

\author{
DAVID AMOS \\ Texas A\&M University \\ e-mail: dave.amos@live.com \\ John Asplund \\ Dalton State College \\ e-mail: jasplund@daltonstate.edu \\ BORIS BRIMKOV \\ Rice University \\ e-mail: boris.brimkov@rice.edu \\ AND \\ RANDY DAVILA \\ University of Johannesburg \\ University of Houston-Downtown \\ e-mail: davilar@uhd.edu
}

\begin{abstract}
In this paper we introduce and study a new graph invariant derived from the degree sequence of a graph $G$, called the sub-k-domination number and denoted $\operatorname{sub}_{k}(G)$. This invariant serves as a generalization of the Slater number; in particular, we show that $\operatorname{sub}_{k}(G)$ is a computationally efficient sharp lower bound on the $k$-domination number of $G$, and improves on several known lower bounds. We also characterize the sub- $k$-domination numbers of several families of graphs, provide structural results on sub- $k$ domination, and explore properties of graphs which are $\operatorname{sub}_{k}(G)$-critical with respect to addition and deletion of vertices and edges.
\end{abstract}

Keywords: Slater number, domination number, sub- $k$-domination number, $k$-domination number, degree sequence index strategy.

2010 Mathematics Subject Classification: 05C69. 


\section{REFERENCES}

[1] Y. Caro and R. Pepper, Degree sequence index strategy, Australas. J. Combin. 59 (2014) 1-23.

[2] Y. Caro and Y. Roditty, A note on the $k$-domination number of a graph, Int. J. Math. Math. Sci. 13 (1990) 205-206.

doi:10.1155/S016117129000031X

[3] E. DeLaViña, C.E. Larson, R. Pepper and B. Waller, Graffiti.pc on the 2-domination number of a graph, Congr. Numer. 203 (2010) 15-32.

[4] W.J. Desormeaux, T.W. Haynes and M.A. Henning, Improved bounds on the domination number of a tree, Discrete Appl. Math. 177 (2014) 88-94. doi:10.1016/j.dam.2014.05.037

[5] M. Dorfling, W.D. Goddard and M.A. Henning, Domination in planar graphs with small diameter II, Ars Combin. 78 (2006) 237-255.

[6] Z. Du and A. Ilić, A proof of the conjecture regarding the sum of domination number and average eccentricity, Discrete Appl. Math. 201 (2016) 105-113. doi:10.1016/j.dam.2015.08.002

[7] O. Favaron, M. Mahěo and J.F. Saclě, On the residue of a graph, J. Graph Theory 15 (1991) 39-64. doi:10.1002/jgt.3190150107

[8] O. Favaron, A. Hansberg and L. Volkmann, On the k-domination and minimum degree in graphs, J. Graph Theory 57 (2008) 33-40. doi:10.1002/jgt.20279

[9] J.F. Fink and M.S. Jacobson, $n$-domination in graphs, Graph Theory with Applications to Algorithms and Computer Science (Kalamazoo, Mich., 1987) 283-300.

[10] M. Gentner and D. Rautenbach, Some comments on the Slater number, (2016). preprint arXiv:1608.04560v1

[11] R. Glebov, A. Liebenau and T. Szabó, On the concentration of the domination number of the random graph, SIAM J. Discrete Math. 29 (2015) 1186-1206. doi:10.1137/12090054X

[12] A. Hansberg, Bounds on the connected $k$-domination number in graphs, Discrete Appl. Math. 158(2010) 1506-1510. doi:10.1016/j.dam.2010.05.021

[13] A. Hansberg and R. Pepper, On k-domination and $j$-independence in graphs, Discrete Appl. Math. 161 (2013) 1472-1480. doi:10.1016/j.dam.2013.02.008

[14] T.W. Haynes, S.T. Hedetniemi and P.J. Slater, Domination in Graphs: Advanced Topics (Marcel Decker, New York, 1998).

[15] T. Haynes, S.T. Hedetniemi and P.J. Slater, Fundamentals of Domination in Graphs (Marcel Decker, New York, 1998). 
[16] M.A. Henning and A. Yeo, Total Domination in Graphs (Springer Monographs in Mathematics, 2013). doi:10.1007/978-1-4614-6525-6

[17] M.S. Jacobson and K. Peters, Complexity questions for $n$-domination and related parameters, Congr. Numer. 68 (1989) 7-22.

[18] A.P. Kazemi, On the total k-domination number of graphs, Discuss. Math. Graph Theory 32 (2012) 419-26. doi:10.7151/dmgt.1616

[19] M. Lemańska, Lower bound on the domination number of a tree, Discuss. Math. Graph Theory 24 (2004) 165-169. doi:10.7151/dmgt.1222

[20] R. Pepper, Binding Independence, PhD Thesis (University of Houston 2004).

[21] R. Pepper, Implications of some observations about the $k$-domination number, Congr. Numer. 206 (2010) 65-71.

[22] D. Rautenbach and L. Volkmar, New bounds on the $k$-domination number and the $k$-tuple domination number, Appl. Math. Lett. 20 (2007) 98-102. doi:10.1016/j.aml.2006.03.006

[23] P.J. Slater, Locating dominating sets and locating-dominating sets, in: Graph Theory, Combinatorics, and Applications, Proceedings of the 7th Quadrennial International Conference on the Theory and Applications of Graphs 2 (1995) 1073-1079.

[24] D. Stevanović, M. Aouchiche and P. Hansen, On the spectral radius of graphs with a given domination number, Linear Algebra Appl. 428 (2008) 1854-1864. doi:10.1016/j.laa.2007.10.024

Received 15 February 2017

Revised 5 February 2018 Accepted 28 February 2018 